\title{
Pulmonary-Vein Stenosis Can Mimic Massive Pulmonary Embolism After Radiofrequency Ablation for Atrial Fibrillation
}

\author{
Wei Wang MD, Jian Ping Zhou MD, Li Qun Wu MD, Gang Gu MD, and Guo Chao Shi MD PhD
}

\section{Introduction}

Pulmonary embolism, a medical emergency for pulmonary physicians, is defined as a blockage of the main artery of the lung or one of its branches by embolism, from many sources, such as thrombus, air, amniotic fluid, tumor, or fat. ${ }^{1}$ A good clinician, especially a pulmonary or critical care physician, should consider the possible diagnosis of pulmonary embolism in a highly suspected patient, since prompt diagnosis and treatment can dramatically reduce the morbidity and mortality of this disease. Because of the nonspecific signs and symptoms of pulmonary embolism, imaging is the key to diagnosis. Computed tomography pulmonary angiography (CTPA) has high sensitivity and specificity for pulmonary embolism and is the accepted standard in pulmonary embolism diagnosis. ${ }^{2,3}$ Combined application of CTPA and ventilation-perfusion (V/Q்) lung scan provides a definite diagnosis in $90-99 \%$ of suspected patients. ${ }^{1}$ However, some CTPA findings can lead to a false-positive diagnosis of pulmonary embolism. ${ }^{4}$ We report a patient who was misdiagnosed with pulmonary embolism after radiofrequency-catheter ablation for atrial fibrillation. Both CTPA and V/Q scan indicated a filling defect in the left main pulmonary artery, which was interpreted as a massive pulmonary embolism, but pulmonary angiography revealed that the true diagnosis was pulmonary-vein stenosis.

Drs Wang, Wu, and Gu MD are affiliated with the Department of Cardiology; and Drs Zhou and Shi are affiliated with the Department of Respiratory Diseases, Rui Jin Hospital, Shanghai Jiaotong University School of Medicine, Shanghai, People's Republic of China.

The authors have disclosed no conflicts of interest.

Correspondence: Guo Chao Shi MD PhD, Department of Respiratory Diseases, Rui Jin Hospital, 197 Rui Jin Road II, Shanghai 200025 People's Republic of China. E-mail: shiguochao@hotmail.com.

DOI: $10.4187 /$ respcare.00975
Case Summary

A 65-year-old female presented with hemoptysis, 8 months after radiofrequency-catheter ablation for atrial fibrillation. The hemoptysis was about $10 \mathrm{~mL}$ of bright red blood per day. She had slight fatigue and shortness of breath on exertion, but no chest pain, syncope, fever, or yellow sputum expectoration. Physical examination found oral temperature $36.8^{\circ} \mathrm{C}$, blood pressure $125 / 85 \mathrm{~mm} \mathrm{Hg}$, heart rate 80 beats/min, respiratory rate 16 breaths/min, and $\mathrm{S}_{\mathrm{pO}_{2}} 98 \%$ on room air. Lung auscultation revealed normal breath sounds and no crackles or rhonchi. Her heart rate was normal with a regular rhythm, normal S1 and S2 heart sounds, and no murmurs, rubs, or gallops.

Laboratory analyses included complete blood count, disseminated intravascular coagulation, D-dimer, liver, and renal function tests, and basic metabolic panel, which were all within normal range. Bacterial and fungal culture of sputum and blood samples were negative. CTPA showed left-pulmonary-vein stenosis and filling defect in the left main pulmonary artery (Fig. 1). V/Q scan also showed, on several views, a severe reduction in perfusion to the left lobe, and slight reduction in ventilation, without obvious defects (Fig. 2). The $\dot{V} / \dot{Q}$ mismatch on V/Q் scan and the filling defect on CTPA highly suggested massive left-lung pulmonary embolism to the pulmonary physicians, cardiologists, and radiologists who examined the images. Interestingly, the patient had no pulmonary-embolism risk factors and had been on anticoagulation therapy after the radiofrequency ablation, so pulmonary angiography was then done, which showed the left and right pulmonary arteries and their branches clearly without any filling defects (Fig. 3).

\section{Discussion}

Pulmonary embolism is a common, emergency, and potentially lethal condition, and massive pulmonary embolism accounts for about $4 \%$ of pulmonary embolism cases. ${ }^{5,6}$ Combined CTPA and V/Q scan has a high sensitivity for detecting pulmonary embolism, ${ }^{1}$ and CTPA is the accepted standard for pulmonary embolism diagnosis. ${ }^{7,8}$ However, 


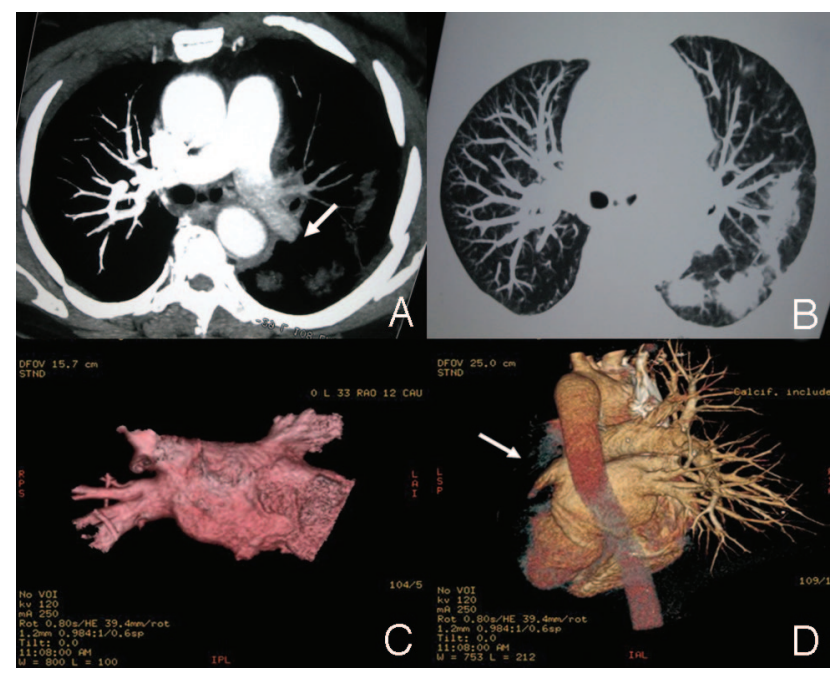

Fig. 1. Spiral computed tomography pulmonary angiogram 8 months after radiofrequency ablation. A: Filling defect in left pulmonary artery (arrow). B: Vague opacity in the lung window of the same section as A, which should be the manifestation of venous congestion; however, there might also have been infection following blood congestion. C: Multiplanar rendering of the computed tomogram does not show the left pulmonary vein or its branches. D: Multiplanar rendering of the computed tomogram does not show the left pulmonary vein or artery (arrow).

reliance on CTPA and $\dot{\mathrm{V}} / \mathrm{Q}$ scan alone can lead to a falsepositive diagnosis of pulmonary embolism. ${ }^{4}$ This teaching case is an example in point.

Differentiating between severe pulmonary-vein stenosis and pulmonary embolism is complicated and may confuse inexperienced pulmonary physicians and radiologists. Pulmonary-vein stenosis and pulmonary embolism share similar nonspecific symptoms, and these patients range from asymptomatic to highly symptomatic, with cough, shortness of breath, chest pain, fatigue, and/or hemoptysis. ${ }^{7,9}$ Also, $\dot{V} / \mathbf{Q}$ mismatch or perfusion defect of a certain lobe on $\dot{V} / \dot{Q}$ scan occurs in $80 \%$ of pulmonary-vein-stenosis patients, and a higher percentage in pulmonary-embolism patients. ${ }^{10-14}$ Thus, it is hard to distinguish between pulmonary-vein stenosis and pulmonary embolism based on symptoms and $\dot{V} / \dot{Q}$ scan. CTPA is more sensitive for differentiation because it shows the positions of vein stenosis and pulmonary embolus separately. ${ }^{2-4,13}$ However, with our patient the massive filling defect on CTPA misled the clinicians and radiologists to the misdiagnosis of pulmonary embolism. Actually, pulmonary-vein stenosis triggers pulmonary congestion, increases the capillary wedge pressure, and ultimately slows the pulmonary arterial flow. Post-capillary obstruction decreases or prevents the circulation of radio-labeled particles and contrast to the affected lung regions and results in Vं/Q mismatch on Vं/Q scan and filling defect on PTCA. Thus, this is not a true filling defect from an embolus, but a slow filling of the contrast.

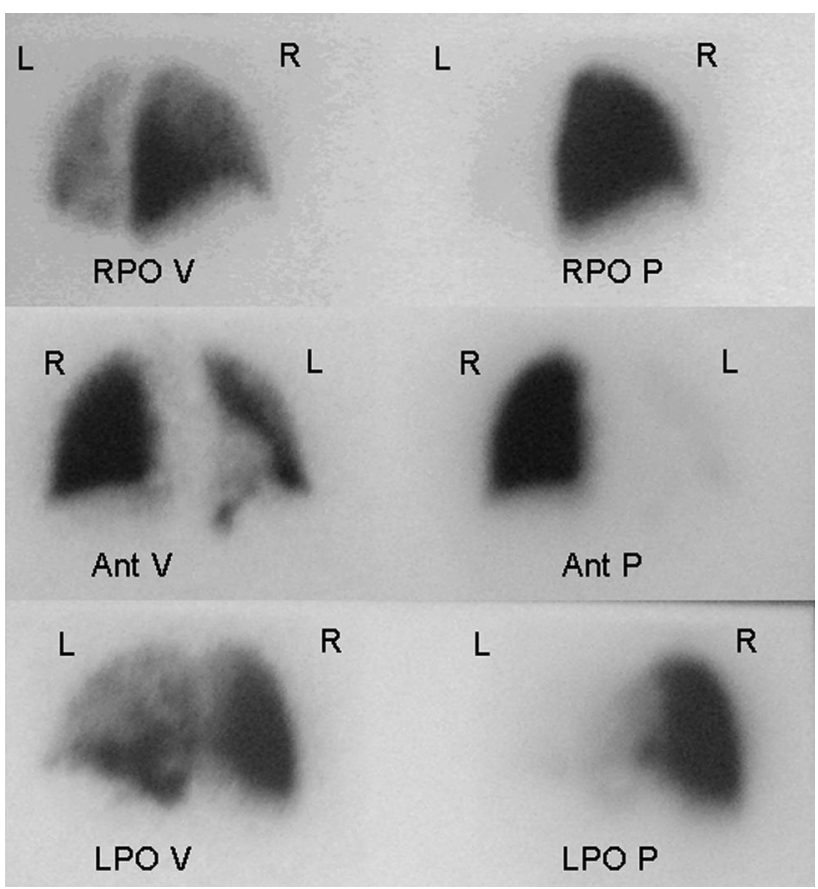

Fig. 2. Radionuclide ventilation-perfusion scintigraphy images show a severe reduction in perfusion to the left lobe, and slight reduction in ventilation, without obvious defects. The ventilation-perfusion mismatch highly suggested pulmonary embolism in the left lung.

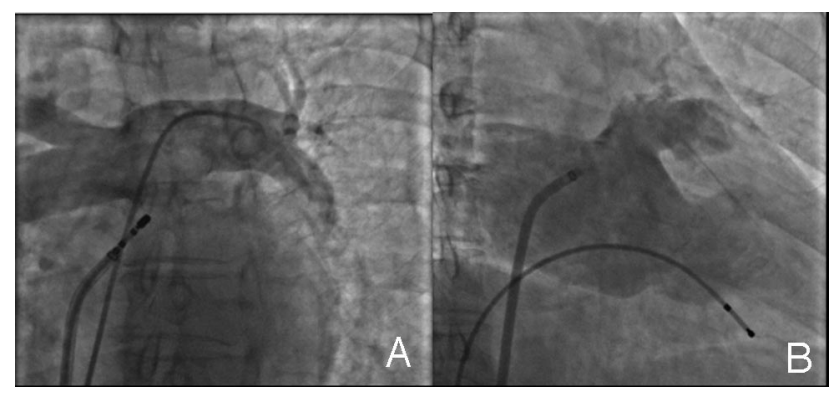

Fig. 3. Angiogram shows (A) complete filling of the pulmonary artery system, without defects, and (B) severe stenosis in the left pulmonary vein.

Lack of experience in recognizing the typical morphology of a pulmonary embolus is another cause of misdiagnosis. The diagnosis of pulmonary embolism on CTPA is based on finding filling defects within the contrast-enhanced lumens of the pulmonary arteries or direct observation of an intra-luminal clot. 15,16 Typical manifestations of embolus on CT include the "lifesaver" sign perpendicular to the long axis of the vessel, the "railway track" sign on longitudinal images of the vessel, and the Westermark sign, which refers to peripheral wedge-shaped regions of ground-glass opacity or consolidation. ${ }^{17-19}$ However, in our patient the sharp artery cutoff on CTPA (arrow in Fig. 1A) was not typical of an acute pulmonary embolus. Also, there was a vague opacity in the left lung on the 
CTPA lung window, and that should be the manifestation of venous congestion (see Fig. 1B). If the patient had massive pulmonary embolism, the CTPA lung window should have shown ischemia manifestations such as oligemia or decrease in vessel diameter and reduction of flow rate, but no evidence of venous congestion. Therefore, CTPA suggested that the occlusion was not in the pulmonary artery where the filling defect was located, but in the post-capillary pulmonary vein. Pulmonary-vein stenosis might also present with chest-radiograph findings of pleural effusion and diffuse interlobular septal thickening. ${ }^{20}$ Further, the patient had no severe symptoms such as shortness of breath at rest, low oxygen saturation, or shock. All these details support the finding that this massive filling defect indicated slow flow but not an embolus.

The complications of radiofrequency-catheter ablation, which is widely used to treat atrial fibrillation, include thromboembolism and pulmonary-vein stenosis. ${ }^{13,21} \mathrm{Com}-$ plete pulmonary-vein occlusion may lead to pulmonaryvein thrombosis or even infarction, with substantial venous congestion in the affected lobe. ${ }^{22}$ Symptoms of pulmonary-vein thrombosis usually arise several months after radiofrequency-catheter ablation, and are less acute than those of pulmonary-artery embolism. Characteristic CT manifestations of pulmonary-vein thrombosis include venous congestion and alveolar hemorrhage, which are similar to the manifestations of pulmonary-vein stenosis. There have been no reports of pulmonary-vein stenosis or pulmonary-vein thrombosis accompanied by pulmonaryartery embolism following radiofrequency-catheter ablation, so more consideration and evidence of arterial embolus should be present before making a diagnosis of pulmonary-vein stenosis accompanied by pulmonary embolism.

In addition to pulmonary-vein stenosis (as in our patient), there are other possibilities for false-positive filling defect on CTPA and Vं/Q scan that can lead to an incorrect diagnosis of pulmonary embolism. ${ }^{23}$ Extra-luminal compression of the pulmonary vasculature from bronchogenic carcinoma, mediastinal mass, aortic aneurysm, sickle cell disease, and idiopathic pulmonary fibrosis are the most common possibilities. ${ }^{24-28}$ Intraluminal obstruction of the pulmonary vasculature by a primary tumor, vasculitis, or a congenital vascular abnormality can also lead to a misdiagnosis. ${ }^{29}$ Some of the above causes are related to the slowing of pulmonary circulation, whereas others directly mimic in-site embolus of pulmonary circulation.

\section{Teaching Points}

This teaching case demonstrates the risk of misinterpretation of filling defect on CTPA and V/Q scan in a patient with severe pulmonary-vein stenosis after radiofrequency ablation. The CTPA and V/Q scan findings mimicked mas- sive pulmonary embolism. Slow flow, luminal compression by soft tissues, and intraluminal obstructions all contribute to the filling defects on CTPA, so extreme caution is warranted when interpreting filling defects on spiral CT and $\dot{V} / \dot{Q}$ scan in patients with such conditions. Other imaging findings that suggest venous congestion (eg, vague opacity on CT lung window, interlobular septal thickening, and pleural effusion on radiograph), and detailed medical history and careful physical examination can yield clues to the right diagnosis.

\section{REFERENCES}

1. Horlander KT, Mannino DM, Leeper KV. Pulmonary embolism mortality in the United States, 1979-1998: an analysis using multiplecause mortality data. Arch Intern Med 2003;163(14):1711-1717.

2. Mos IC, Klok FA, Kroft LJ, A De Roos A, Dekkers OM, Huisman MV. Safety of ruling out acute pulmonary embolism by normal computed tomography pulmonary angiography in patients with an indication for computed tomography: systematic review and metaanalysis. J Thromb Haemost 2009;7(9):1491-1498.

3. Cronin P, Weg JG, Kazerooni EA. The role of multidetector computed tomography angiography for the diagnosis of pulmonary embolism. Semin Nucl Med 2008;38(6):418-431.

4. Ranji SR, Shojania KG, Trowbridge RL, Auerbach AD. Impact of reliance on CT pulmonary angiography on diagnosis of pulmonary embolism: a Bayesian analysis. J Hosp Med 2006;1(2):81-87.

5. Goldhaber SZ, Visani L, De Rosa M. Acute pulmonary embolism: clinical outcomes in the International Cooperative Pulmonary Embolism Registry (ICOPER). Lancet 1999;353(9162):1386-1389.

6. Kucher N, Rossi E, De Rosa M, Goldhaber SZ. Massive pulmonary embolism. Circulation 2006;113(4):577-582.

7. Tapson VF. Acute pulmonary embolism. N Engl J Med 2008;358(10): 1037-1052.

8. Wood KE. Major pulmonary embolism: review of a pathophysiologic approach to the golden hour of hemodynamically significant pulmonary embolism. Chest 2002;121(3):877-905.

9. Amin R, Kwon S, Moayedi Y, Sweezey N. Pulmonary vein stenosis: case report and literature review. Can Respir J 2009;16(6):e77-e80.

10. Nguyen BD. AJR Teaching File: dyspnea following surgical repair of partial anomalous venous return. AJR Am J Roentgenol 2007; 189(3 Suppl):S26-S28.

11. Zophel K, Bacher-Stier C, Pinkert J, Kropp J. Ventilation/perfusion lung scintigraphy: what is still needed? A review considering technetium-99m-labeled macro-aggregates of albumin. Ann Nucl Med 2009;23(1):1-16.

12. Freeman LM, Haramati LB. V/Q scintigraphy: alive, well and equal to the challenge of CT angiography. Eur J Nucl Med Mol Imaging 2009;36(3):499-504.

13. Packer DL, Keelan P, Munger TM, Breen JF, Asirvatham S, Peterson LA, et al. Clinical presentation, investigation, and management of pulmonary vein stenosis complicating ablation for atrial fibrillation. Circulation 2005;111(5):546-554.

14. Nanthakumar K, Mountz JM, Plumb VJ, Epstein AE, Kay GN. Functional assessment of pulmonary vein stenosis using radionuclide ventilation/perfusion imaging. Chest 2004;126(2):645-651.

15. Blachere H, Latrabe V, Montaudon M, Valli N, Couffinhal T, Raherisson C, et al. Pulmonary embolism revealed on helical CT angiography: comparison with ventilation-perfusion radionuclide lung scanning. AJR Am J Roentgenol 2000;174(4):1041-1047.

16. Remy-Jardin M, Remy J, Baghaie F, Fribourg M, Artaud D, Duhamel A. Clinical value of thin collimation in the diagnostic workup 


\section{Pulmonary-Vein Stenosis Can Mimic Massive Pulmonary Embolism}

of pulmonary embolism. AJR Am J Roentgenol 2000;175(2):407411.

17. Zhang L, Deng X, Wang Y, Zhai R. Precision pulmonary angiography for the diagnosis of massive and small pulmonary thromboembolism using dual-slice spiral computed tomography. Zhonghua Jie He He Hu Xi Za Zhi 2002;25(7):412-416.

18. Wittram C, Maher MM, Yoo AJ, Kalra MK, Shepard JA, McLoud TC. CT angiography of pulmonary embolism: diagnostic criteria and causes of misdiagnosis. Radiographics 2004;24(5):1219-1238.

19. Ray JG. Westermark sign and suspected pulmonary embolism (letter). Can J Cardiol 2003;19(3):317.

20. Saad EB, Marrouche NF, Saad CP, Ha E, Bash D, White RD, et al. Pulmonary vein stenosis after catheter ablation of atrial fibrillation: emergence of a new clinical syndrome. Ann Intern Med 2003;138(8): 634-638.

21. Bertaglia E, Zoppo F, Tondo C, Colella A, Mantovan R, Senatore G, et al. Early complications of pulmonary vein catheter ablation for atrial fibrillation: a multicenter prospective registry on procedural safety. Heart Rhythm 2007;4(10):1265-1271.

22. Gleeson TG, Cheyne I, English JC, Quadri SM, Leipsic JA. A 50-yearold woman with a history of atrial fibrillation presents with acute dyspnea and pleuritic chest pain. Chest 2010;137(5):1225-1230.

23. Zhou C, Chan HP, Patel S, Cascade PN, Sahiner B, Hadjiiski LM, et al. Preliminary investigation of computer-aided detection of pulmo- nary embolism in three-dimensional computed tomography pulmonary angiography images. Acad Radiol 2005;12(6):782-792.

24. Stanley DC, Cho SR, Tisnado J, Vines FS, Coyne SS. Pulmonary arteriography in patients with hilar or mediastinal masses and lung scans suggesting pulmonary embolism. South Med J 1981;74(8): 960-967.

25. Jong I, Taubman K, Schlicht S. Rapid autolysis of pulmonary emboli: a potential cause for apparent false-positive V/Q scintigraphy on non-contemporaneous CTPA findings. Clin Nucl Med 2005; 30(12):818-819.

26. Abikhzer G, Zand KR, Stern J, Laufer J. False positive high probability V/Q scan due to malignant obstruction of both pulmonary vein and artery. Clin Nucl Med 2009;34(6):367-370.

27. Wong KK, Gruenewald SM, Larcos G. Ventilation-perfusion mismatch resulting from iatrogenic pulmonary vein stenosis after radiofrequency ablation: a case report. Clin Cardiol 2009;32(11):E67E70.

28. Makhija Z, Murgatroyd F, Gall N, Marrinan MT, Deshpande R, Desai SR. Fibrosing mediastinitis and occlusion of pulmonary veins after radiofrequency ablation. Ann Thorac Surg 2009;88(5):16741676.

29. Armas RR. False-positive V/Q scan mimicking massive pulmonary embolism. Clin Nucl Med 1992;17(1):34-35. 